\title{
Versatility of the Coronal Approach in Maxillofacial Surgery
}

\author{
Suhas S Godhi, Pankaj Kukreja, Vishal Singh, Sonia Goyal
}

\section{ABSTRACT}

The coronal approach, also known as bi-temporal approach, is one of the most versatile surgical approaches to the zygomatic arch and the mid-facial skeleton. Excellent access is gained to the mid-face by this approach, and it also has a very less number of complications. It is esthetically also excellent, because most of the surgical scar is hidden within the hairline. When the incision is extended into the preauricular area, the surgical scar is inconspicuous. In this article, we attempt to describe the approach, and present our own experience in using it at our centre.

\section{Contact Author}

Dr. Suhas S. Godhi

E-mail :drgodhi@yahoo.com

Key words: Coronal incision, scalp, naso-ethmoidal complex, frontal bone.

$\mathrm{O}$ $\mathrm{f}$ the various approaches used in the treatment of facial lesions and injuries, the coronal approach, popularized by Tessier, is one of the most versatile of all. Many authors have recommended this approach for midface surgery.(1-6) Through this one surgical approach, it is possible to expose the zygoma, the zygomatic arch, the temporomandibular joint, the sub condylar region, the medial, superior lateral, and part of the inferior rim and walls, the nasal bones, the frontal bone and the calvaria.(7)

\section{Indications}

- Access to frontal sinus, nasal root, nasoethmoid compartment, superior orbits, and zygomatic arch for treatment of pathological and/or traumatic lesions.

- Baldness or a strong family history of male pattern baldness is a relative contraindication to this approach

\section{Contraindications}

Approach to infratemporal fossa, lateral orbital rim, and lateral skull base for treatment of pathological and/or traumatic lesions.

\section{Brief Surgical Anatomy}

The layers of the scalp include from superficial to deep: skin, subcutaneous tissue, galea or frontalis muscle, subgaleal fascia, and the periosteum, although, over the temporalis muscle, the layers of soft tissue are more complicated. Above the temporal line of fusion, which is at the level of the superior orbital rim and delineates the superior aspect of the temporal fat pad, the layers include: skin, subcutaneous tissue, temporoparietal fascia (facial nerve, and the superficial temporal artery run in this layer), deep temporal fascia, temporalis muscle, periosteum. Below the temporal line of fusion the layers include: skin, subcutaneous tissue, temporoparietal fascia, superficial layer of the deep temporal fascia, temporal fat pad (middle temporal artery runs in this pad), deep layer of the deep temporal fascia, temporalis muscle, periosteum.

For males, the emphasis appropriately focuses on the status of the hairline. In some cases of mild male pattern baldness, the incision may be placed posteriorly to hide it in the remaining hair. The patient should be aware that the incision may become visible if hairline recession continues. It must be ensured that the planned incision will afford adequate exposure for the planned procedure.

\section{Overview of the Technique}

The face and entire scalp should be prepared and draped. The hair is washed with Betadine solution.A towel is stapled to the scalp behind the anticipated location of the incision. This should be stapled along the lateral head down to the region of the mastoids behind helical root. tarsorrhaphy sutures may be placed to protect the eyes. Rubber bands may be used to secure hair. Depending on the indication for surgery, the patient will either have an oral endotracheal intubation or tracheostomy. 
The standard coronal incision runs from helical crus to helical crus approximately 2 to $3 \mathrm{~cm}$ posterior to the hairline. The incision line should follow a geometric broken line type of pattern. This will minimize visibility of the incision, particularly when the hair is wet. The initial incision is carried down to the level of the supra periosteum(loose areolar tissue). If the incision is being used as an approach to the frontal sinus, the dissection plane changes to the subperiosteal plane above the frontal sinus on frontal bone. If the access is for upper central facial surgery the dissection changes to the subperiosteal plane approximately 2 $\mathrm{cm}$ above the supraorbital rim. Laterally over the temporalis muscle, the plane of dissection should be deep to the temporoparietal fascia, just above the deep temporal fascia. This plane will allow elevation of the frontal branch of the facial nerve with the coronal flap. If access to the zygoma is required; the superficial layer of the deep temporal fascia is opened just above the arch. If this plane (temporal fat pad) is entered more superiorly, bleeding from the middle temporal artery will be encountered, and atrophy of the fat in this pad will be more pronounced after surgery. Extended access to the upper orbits and nasoethmoid areas requires release of the supraorbital neurovascular bundle that is then reflected anteriorly with the coronal flap. The inferior aspect of the short bony foramen through which this neurovascular bundle occasionally passes is simply removed with a downward tap of the osteotome. Closure of the incision is in two layers.

\section{Steps for a Coronal flap}

- Scalp preparation

- Placing the incision to the supraperiosteum level

- Reflection of the flap supra periosteally,laterally and inferiorly

- Periosteal incision $2 \mathrm{~cm}$ above supra orbital margin \& release of the supra-orbital neurovascular bundle

- Operative procedure

- Closure in layers

- Placement of vacuum drain

- Post-operative care

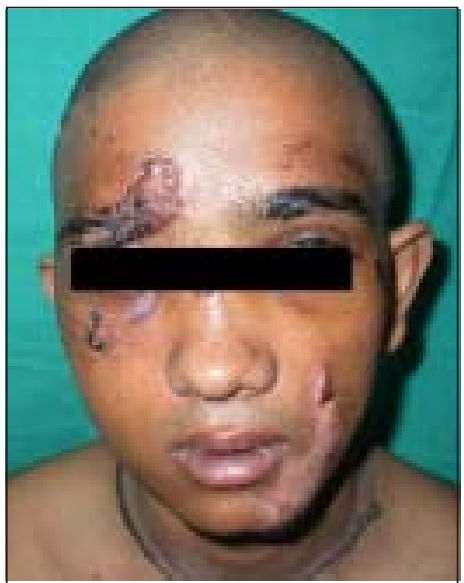

Fig.1 Pre operative frontal view of the patient

\section{Potential Complications}

- Infection

- Hematoma requiring evacuation

- Alopecia along incision line

- Visible scar

- Injury to frontal branch of facial nerve

- Numbness of the flap tissue if supraorbital or supratrochlear nerves are injured

- Anesthesia posterior to the incision.

\section{Case Reports \\ Case 1}

A 20 year old patient riding a motor cycle collided with other motor cycle. He sustained a head injury with initial loss of consciousness for a few minutes and severe naso-ethmoidal fracture. The patient reported to our department one month after the injury and wanted correction of facial deformity following trauma. On examination, there was depressed area in frontal bone, glabella and nasal bridge. The bridge of the nose was deviated towards right side with increased intercanthal distance.First, the reduction of nasal bone was performed. The bicoronal flap was raised by dissection along the plane, superficial to pericranium. The periosteum was divided just above the supraorbital ridges and the dissection was then completed subperiosteally thus virtually degloving the forehead. The dissection was then carried down over the structure in relation to the bridge of the nose. At exploration via bicoronal approach, the position of the displaced frontal bone was clearly visualized. The flap gave excellent exposure for repair of the defect. The onlay bone gafting was performed. The graft was harvested from right anterior iliac crest. The grafts were secured via $2.0 \mathrm{~mm}$ stainless steel miniplates. When the defect has been repaired the flap was turned back. The cosmetic result was excellent as the scar line was hidden almost totally within the hairline.

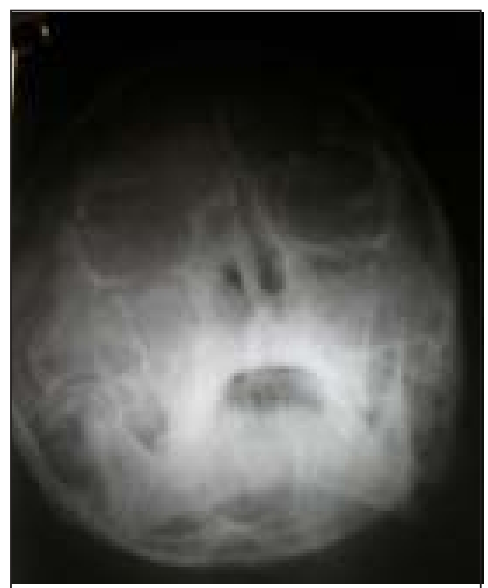

Fig. 2 PNS view

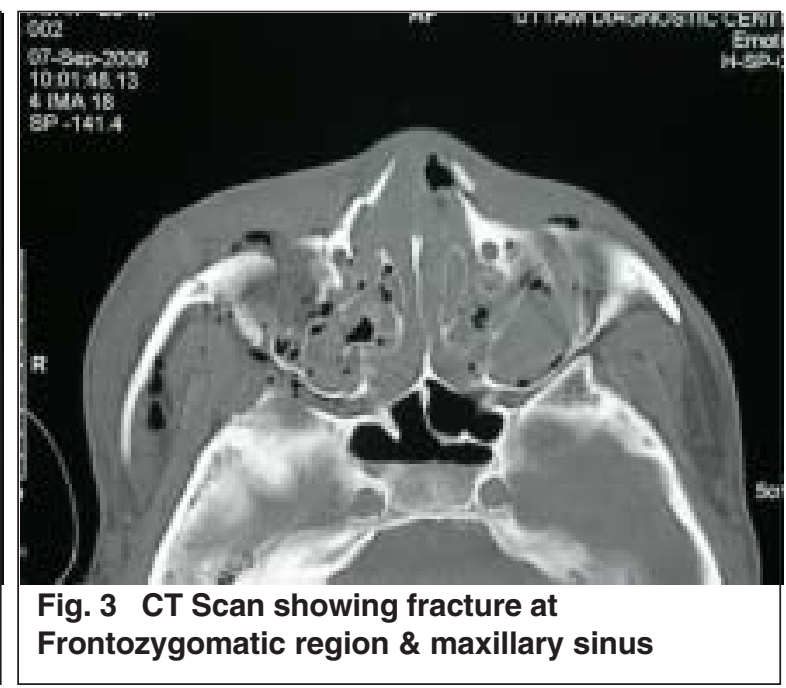




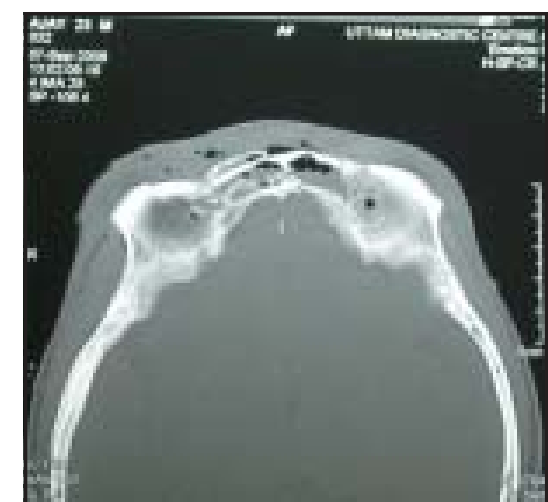

Fig. 4 CT Scan showing fracture at supra orbital \& frontal region

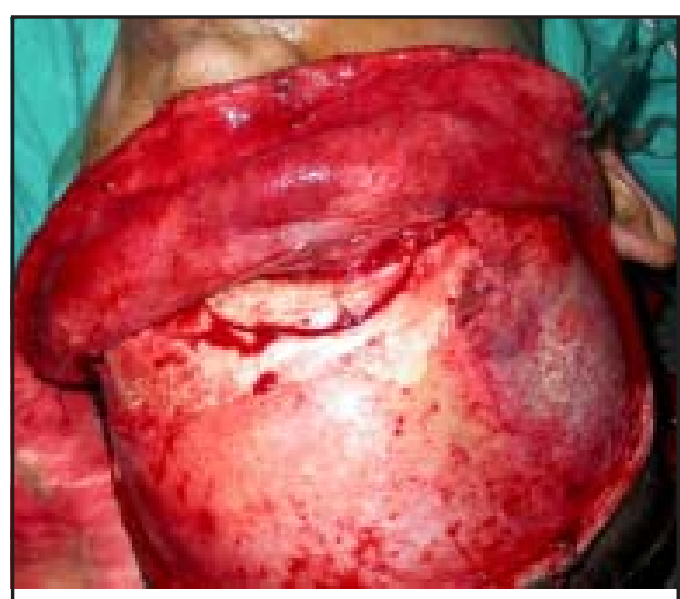

Fig. 7 Exposure of th fracture site

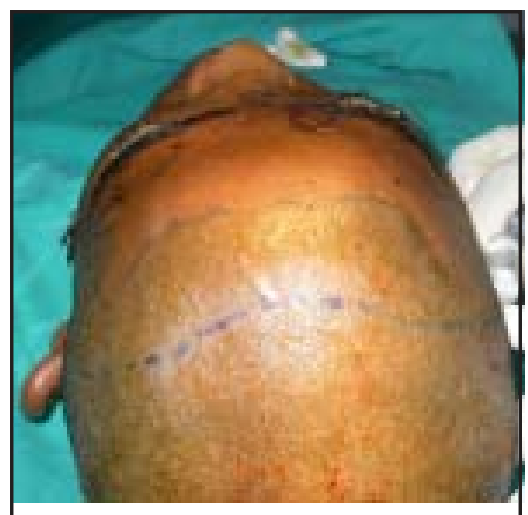

Fig. 5 Coronal incision

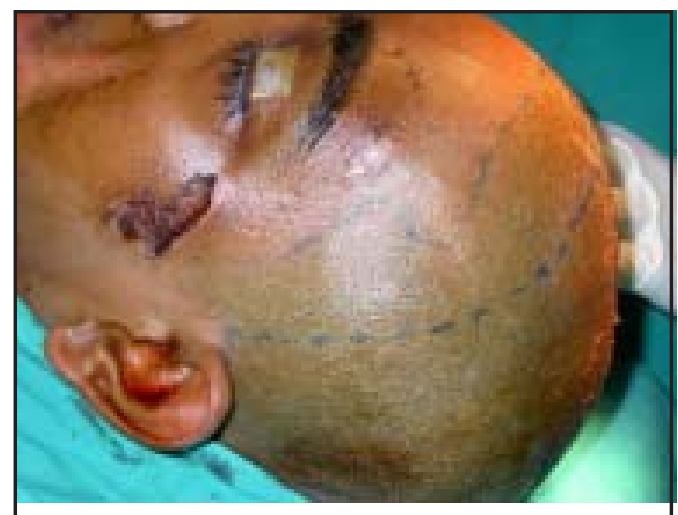

Fig. 6 Coronal incision (Lateral view)

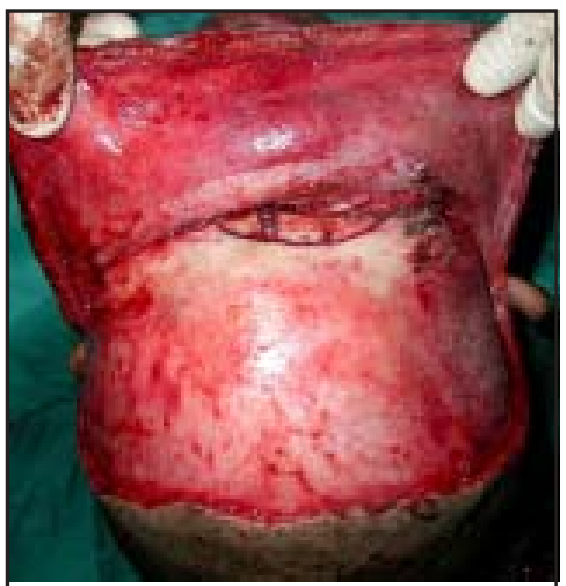

Fig. 8 Frontal, supraorbital \& lefort III fracture reduction \& miniplate osteosynthesis

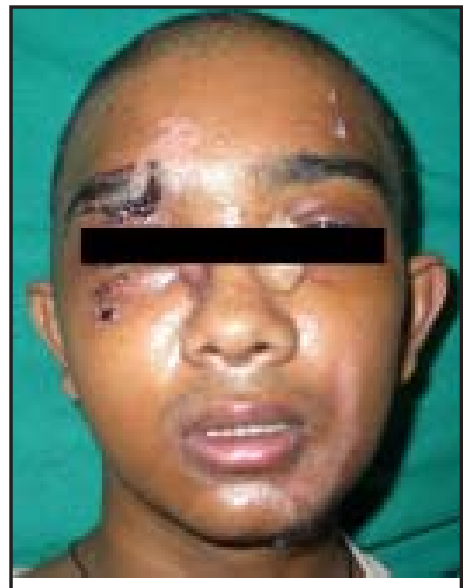

Fig. 9 Immediate post operative view

\section{Case 2}

A 22 year old male was riding a motor cycle and ran into the back of a lorry. He sustained a head injury, a severe nasoethmoidal injury combined with left zygomatic fracture. There was communiation of naso ethmoidal complex with an increased intercantal distance. Immediate repair of the soft tissue lacerations of the eyelids and infraorbital region on the left side was undertaken. A bicoronal flap was then raised. The displaced canthal ligament was repositioned and stabilized by trans-nasal wiring. The depressed fracture of frontal region

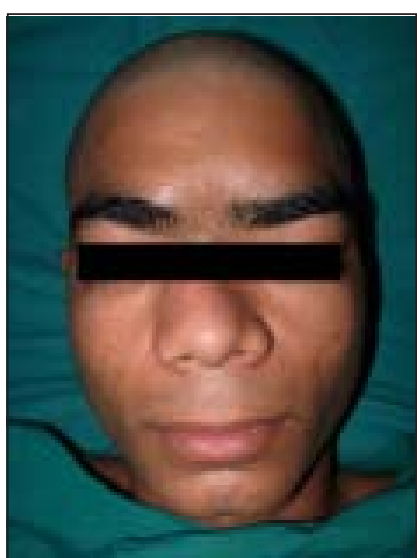

Fig. 10 Pre operative frontal view of the patient

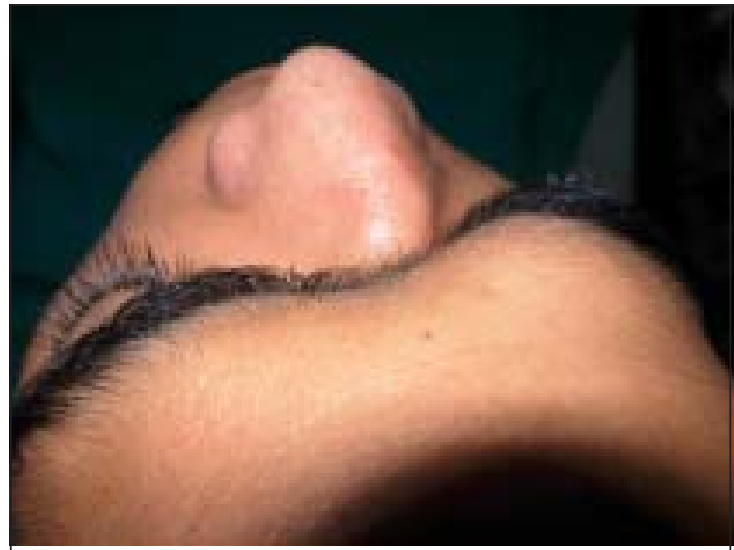

Fig. 11 Pre operative birds eye view showing the deformity

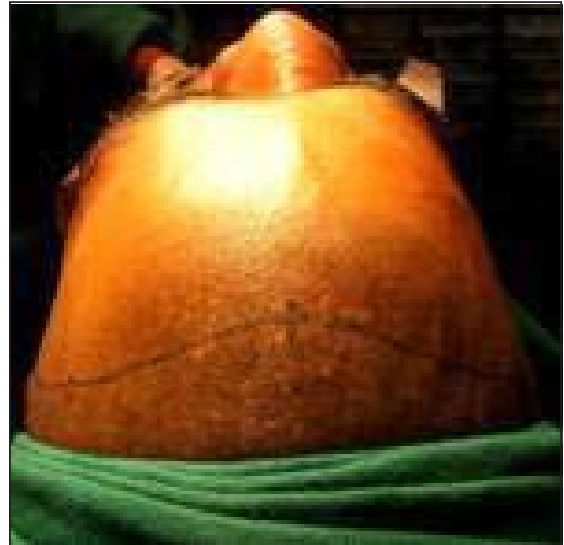

Fig. 12 Marking of coronal incision 

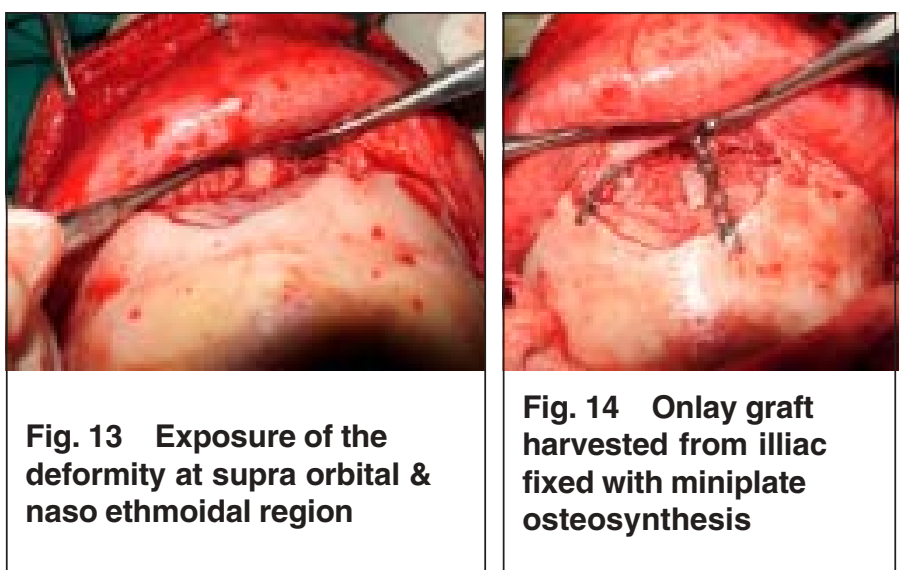

Fig. 14 Onlay graft harvested from illiac fixed with miniplate osteosynthesis
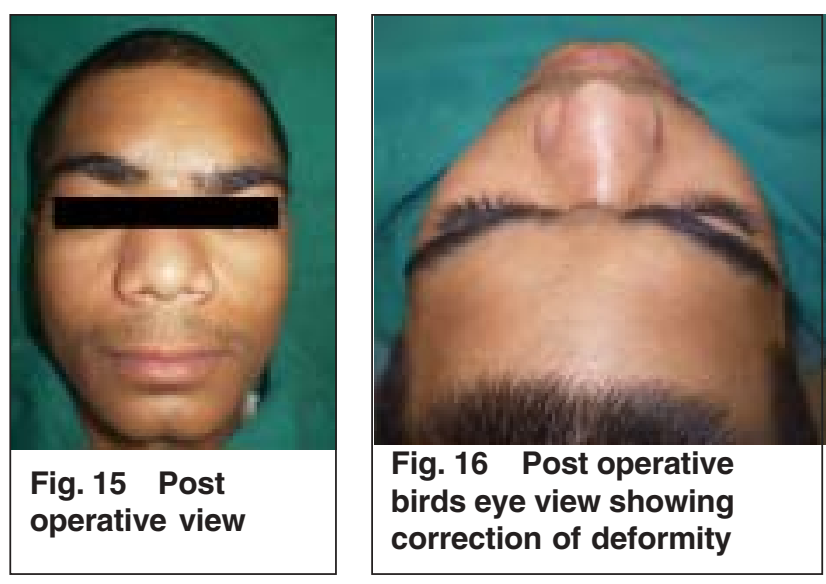

Although condylar fractures of the mandible may be treated by closed reduction and appropriate physiotherapy, open reduction and internal fixation is indicated in specific circumstances. Dunaway and Trott have reported 25 cases of a method of exposure of condylar fractures using an extended bicoronal approach combined with myotomy of the masseter muscle. Acceptable reduction and fixation was achieved in all cases with an early return to function. The incidence of complications was low, with three mild temporary facial palsies which had resolved by the sixth postoperative week and one haematoma beneath the bicoronal scalp flap. A cosmetically acceptable scar was produced in all cases. The excellent surgical exposure and protection of the facial nerve, combined with cosmetically acceptable scars, commend the use of this technique.(10) separation of the hair that can become visible when the hair is wet, such as during swimming. A modification of the incision has been the use of a zig-zag incision instead of a straight incision within the hairline. The zig zag incision helps break up the scar and make it less noticeable, even when the hair is worn short. The major disadvantage of this incision is the increased time needed for closure. (8)

Bicoronal scalp incisions are needed in cranio-facial surgery to gain access to both the skull vault and the face. Bicoronal flaps have a low morbidity even though the incision is extensive. However, with such a large incision there is always the worry of a visible postoperative scar. Various techniques have been described, including a straight incision, bicoronal incisions, a gull-wing or W-shaped incision and the use of six short linear incisions, all trying to decrease the visibility of the postoperative scar. Leach, Rutherford, Likith and Leggate have used a zig-zag incision for our cranio-facial cases and they believe that the hair falls more naturally, especially when wet; therefore, the hair is less likely to part and reveal the scar line. The hair is not shaved, but marked and then parted in a zig-zag fashion using alcohol gel and a comb. The scalp is then prepped and draped in the usual way and the incision made. The galea and skin are closed as separate layers in the usual way. They believe that this technique leads to improved cosmesis with regard to the postoperative scar.(9)
The utilization of the bicoronal scalp flap in craniofacial trauma has proved indispensable in the management of severe craniofacial injuries. It provides vast exposure of such critical structures as the cranium, frontal sinus, orbit and upper midface, compared with that for previous techniques of facial fracture reduction. Although the flap has great utility, severe complications, such as facial nerve injury, diplopia, telecanthus, and scalp necrosis, can occur. Alvi and Carrau have reviewed the surgical anatomy, technique, and indications for the safe utilization of the bicoronal scalp flap approach in the management of craniofacial trauma. (11)

A coronal brow-lifting technique is described by Wojtanowski which uses the coronal approach. This approach represents an ideal aesthetic procedure: It is a technically straightforward operation that yields results which are predictable, natural appearing, and long lasting with minimal morbidity. Although aesthetic surgery on the upper third of the face has received relatively little attention in recent literature, it is an extremely important component of facial aesthetic surgery and often yields results that are quite dramatic. Periorbital rejuvenation, either alone or in conjunction with other aesthetic surgical procedures, offers facial enhancement to younger patients. In addition, older patients may benefit from a "forehead lift" 
performed in conjunction with lower facial surgery. This benefit is derived because lower facial surgery may create facial disharmony by yielding a more youthful lower face while leaving a more mature upper face. In these cases a forehead lift serves to unify the patient's facial appearance. (12)

Hayter and Robertson have discussed the use of the bicoronal flap in the surgical treatment of a case of bilateral coronoid hyperplasia. The surgical approach to large lesions of the coronoid process is discussed in their article. (13)

Atypical gunshot wounds are caused by a diverse set of parameters relating to weapons and ammunition. Agrawal, Malla, Joshi, Kumar and Koirala have reported an atypical mode of gunshot wound produced by a detached rear end of the barrel of a gun following accidental gun fire, and discuss the difficulties in the management. A 36-year-old man presented to their emergency department with an alleged history of injury on the forehead with the rear end of a gun barrel following accidental gunfire while cleaning the nozzle. Since the time of injury, the patient was in an altered sensorium and had weakness on the right side of the body. There was minimal but continuous bleeding from the wound, with extrusion of brain matter. Skull radiograph showed that the rear end of the barrel had entered the left frontal bone, with associated depressed fracture of the frontal bone. The patient underwent a bicoronal, bifrontal craniotomy with a T-shaped extension towards the barrel to facilitate the reflection of the scalp flap and to avoid any movement of the barrel as it might further injure the brain. Necrotic brain, dura and bone pieces were removed. The patient was doing well at follow-up except for mild residual motor deficits. They have illustrated that while working with limited facilities, particularly in underdeveloped countries, a careful clinical assessment, interpretation of available images and a judicious operative approach can help to save the patient. (14)

The use of bicoronal incisions has been sufficiently described in neurosurgery and craniofacial surgery including osteotomies and injuries. This approach provides excellent surgical access for nasal reconstruction with a very low rate of morbidity. Atlan, Jammet, Schmitt-Bernard, Dupoirieux and Souyris have published a series of 11 patients, together with three case reports, and have illustrated the advantages and possible complications of this type of incision.(15)

Maxillofacial surgeons have used the bicoronal flap for nearly three decades to gain access to the craniofacial skeleton. Kerawala, Grime, Stassen and Perry have done a retrospective analysis of 68 bicoronal flaps done over a five-year period and have shown that their incidence of permanent morbidity was low. Although 24 patients (35\%) experienced some form of sensory abnormality immediately after the operation, this persisted for longer than two years in only one. Complete motor recovery occurred by one year in all 15 patients (22\%) who developed postoperative frontalis weakness. Three patients developed male pattern baldness postoperatively, which resulted in exposure of the scar and poor cosmesis. This prompted a cadaveric study in which we assessed the feasibility of modifying the position of the standard bicoronal incision in people who are prone to hair loss. The pivotal point of the bicoronal flap was found to lie at its most inferior aspect. By extending the incision into the skin crease in front of the lobe of the ear it was possible to adjust the anteroposterior position of the bicoronal incision without limiting access to the facial skeleton. They have, therefore, advocated occipitally placed incisions with preauricular extensions in patients who are prone to male pattern baldness. (16)

Earnst, Herzog and Seidl have described the technique of coronal incision in their textbook. A skin incision is made from ear to ear, placed bilaterally preauricular over the temporalis muscle attatchment, running $2 \mathrm{~cm}$ behind the hairline to a point inferior to the tragus. After dissecting the subcutaneous layer, the periosteum is incised. Subperiosteal dissection minimises the postoperative swelling. The Dissection then proceedes beneath the periosteum to the temporal fossa. To avoid injury of the superficial branches of the facial nerve, care must be taken with further incision to strictly dissect against the temporal fascia or in the subfascial plane. The supraorbital nerve is exposed on both the sides within its bony canal, and then the orbits are exposed. In the course of dissection, the entire central midface can be exposed down to the lacrimal sac. If the incision is continued bilaterally to inferior of the tragus, dissection allows exposure of the cartillagenous nasal skeleton. (17)

The bicoronal incision, popularized by Tessier, provides wide access to the orbits, nose, and zygomas as well as the cranium. But blood loss is unavoidable. Park, Kyeong, Ha, and Kim have attempted to reduce the blood loss and the transfusion with tumescent technique. Athors had performed 17 cases of bicoronal incision between June, 2002 and March, 2003. 8 cases of them underwent the procedure with tumescent solution (Group A). 9 cases were done by usual manner (Group B). They analyzed 23 cases undergiong bicoronal incision for the blood loss and the transfusion amount. Intraoperative blood loss, postoperative transfusion was reduced at Group A. And the dissecting was easy. They think that tumescent technique contributes to reduce the bleeding, additionally reduce the postoperative transfusion. (18)

Fractures of the frontal sinus are a relatively common injury presenting to trauma units that deal with craniofacial injuries. Approximately one third of frontal sinus fractures affect the anterior wall alone, with two thirds involving the anterior wall, posterior wall, or frontonasal duct. Isolated posterior wall defects are exceedingly rare. Frontal sinus fracture 
management is still controversial and involves preserving function when feasible or obliterating the sinus and duct, depending on the fracture pattern. In the standard treatment modality of frontal sinus fractures, repair is best performed by way of a coronal approach, which offers excellent access. Most of the frontal sinus fractures deserve this attentive surgical manipulation to prevent late sequelae of infection or mucocele formation. Mavili and Canter, in their article, have presented a case of isolated depressed anterior wall fracture of the frontal sinus that was treated by closed reduction to avoid coronal incision. Anterior wall fracture of the right frontal sinus was diagnosed with preoperative evaluation of three-dimensional CT of a 34-year-old male patient with maxillofacial trauma. The anterior wall fracture was reduced by traction of two percutaneously applied screws to the depressed fragments. Accurate reduction was obtained, and neither recurrent displacement nor infection was observed during the followup period of 3 months. The screws were removed in the clinical setting without difficulty. Although percutaneous reduction of noncomminuted anterior wall frontal sinus fractures has limited indications, it has its own advantages over open techniques. This method is a less-invasive technique and can be performed without problem in selected cases. Their technique is not suitable for complex fractures of the frontal sinus. (19)

\section{Conclusion}

The coronal flap is a well recognized technique for exposing the skull and upper face. The coronal flap has recently become a preferred approach for access to the craniofacial skeleton and orbit. The variety of cases in which it has proven indispensable includes craniofacial reconstruction, facial trauma, and tumor resection. This method of exposure has become particularly useful with increased indications for rigid internal fixation and primary bone grafting in the management of complex facial fractures. We have attempted in this article to review the indications, benefits and potential complications of the coronal approach, with a brief description of the anatomy and technique.

\section{THE AUTHORS}

\section{Dr. Suhas S. Godhi}

M.D.S

Professor, Department of Oral \& Maxillofacial Surgery

I.T.S Centre for Dental Studies \& Research

Delhi-Meerut Road, Muradnagar

Ghaziabad - 201206 (U.P)

Cell: 0-9899450488

E-mail : drgodhi@yahoo.com

\section{Dr. Pankaj Kukreja}

M.D.S

Senior lecturer, Department of Oral \& Maxillofacial Surgery

I.T.S Centre for Dental Studies \& Research

Delhi-Meerut Road, Muradnagar
Ghaziabad - 201206 (U.P)

Cell: 0-9999998558

E-mail : kukrejapankaj@rediffmail.com

\section{Dr. Vishal Singh}

M.D.S

Associate Professor, Department of Oral \& Maxillofacial Surgery,

Himachal Dental College, Sundarnagar,

Himachal Pradesh.

Cell: 0-9805487602

\section{Dr. Sonia Goyal}

M.D.S

Ex-Associate Professor, Department of Oral \& Maxillofacial Surgery I.T.S Centre for Dental Studies \& Research

Delhi-Meerut Road, Muradnagar

Ghaziabad - 201206 (U.P)

\section{References}

1. Jones WD, Whitaker LA, Mutagh F. Applications of reconstructive craniofacial techniques to acute craniofacial trauma. J Trauma 1977; 17:339

2. Gruss JS, Makinnin SE. Complex maxillary fractures: role of buttress reconstruction and immediate bone grafts. Plast Reconstr Surg 1952;9:276

3. Manson PN, Crawley WA, Yaremchuk MJ, Rochman GM, Hoopes JE, French JH Jr., Midface Fractures: Advantages of Immediate Extended Open Reduction and Bone Grafting. Plast Reconstr Surg 1985;72:1-10.

4. Stanley RB. The zygomatic arch as a guide to reconstruction of comminuted malar fractures. Arch Otolaryngotol Head Neck Surg 1989;115:1459.

5. Gruss JS. Complex nasoethmioid orbitaland midfacial fractures: role of craniofacial surgical techniques and immediate bone grafting. Ann Plast Surg 1986;17:377

6. Shumrick KA. Recent advances and trends in management of maxillofacial and frontal trauma. Fac Plast Surg 1993;9;16

7. Fonseca RJ, Walker RV, Betts NJ, Barber HD, Powers MP. Oral and maxillofacial trauma, $3^{\text {rd }}$ ed, 2005, Elsevier saunders. 338-339.

8. Ellis E III, Zide MF. Surgical approach to the facial skeleton. $2^{\text {nd }}$ ed 2005. Lippincot Williams and Wilkins 91-92

9. Leach P, Rutherford S, Likhith A, Leggate J. Zig-zag bicoronal scalp incision for cranio-facial cases in paediatric neurosurgery. Childs Nerv Syst 2004;20(7):483-4.

10. Dunaway DJ, Trott JA. Open reduction and internal fixation of condylar fractures via an extended bicoronal approach with a masseteric myotomy. J Plast Reconstruct Aesthet Surg 1996; 49(2);78

11. Alvi A, Carrau RL. The bi-coronal flap approach in craniomaxillofacial trauma. J Craniomaxillofac Trauma 1996;2(2); 40-55.

12. Wojtanowski MH. Bicoronal Forehead Lift. Aesth Plast Surg 1994; 18:33-39.

13. Hayter JP, Robertson JM. Surgical access to bilateral coronoid hyperplasia using the bicoronal flap. Br J Oral Maxillofac Surg 1989;27(6);487-493

14. Agrawal A, Malla G, Joshi S, Kumar A, Koirala S. Unusual mode of firearm injury from the recoiled rear end of a gun barrel. Singapore Med J 2008;49(9): e238

15. Atlan G, Jammet P, Schmitt-Bernard CF, Dupoirieux L, Souyris F. Bicoronal incision for nasal bone grafting. Int $J$ Oral Maxillofac Surg. 1994;23(1):2-5

16. Kerawala CJ, Grime RJ, Stassen LFA, Perry M. The bicoronal flap (craniofacial access): an audit of morbidity and a proposed surgical modification in male pattern baldness $\mathrm{Br} \mathrm{J}$ Oral Maxillofac Surg 2000;38(5);441-444.

17. Earnst A, Herzog M, Seidl RO. Head and neck trauma, an interdisciplinary approach. $1^{\text {st }}$ ed, Thieme $2006 ; 140$.

18. Park JH, Kyeong HS, Ha DH, Kim DI. The Use of Tumescent Solution in Bicoronal Incision. J Korean Cleft Palate-Craniofac Assoc 2003;4(2):178-182.

19. Mavili ME, Canter HI. Closed Treatment of Frontal Sinus Fracture With Percutaneous Screw Reduction. J Craniofac Surg 2007;18(2):415-419. 